\title{
Historia económica en el Antropoceno: cuatro modelos
}

\author{
JULIA ADENEY THOMAS
}

Bajo la amenaza global del Antropoceno, la historia ambiental y la historia económica se conjugan para alcanzar mayor entendimiento de la situación actual. Este nuevo campo de la "historia eco-económica" rastrea el impacto ecológico del sorprendente aumento en la productividad de la economía mundial en los últimos dos siglos. La naturaleza ya no es vista como una externalidad y el daño a los recursos no renovables ya no se minimiza. He identificado cuatro modelos eco-económicos básicos. El retromodernista nos hace retornar a un mundo eurocéntrico, tanto para los orígenes del problema como para sus soluciones. Tres modelos más convincentes, modernidad de doble capa, modernidades paralelas y enfoques multiescala, amplían la comprensión de cómo llegamos a esta coyuntura catastrófica y lo que podríamos hacer al respecto.

PALABRAS ClAVE: Antropoceno, cambio climático, historia económica, historia ambiental, Asia, Japón, China, África, desarrollo

\section{Economic History in the Anthropocene: Four Models}

Under the global threat of the Anthropocene, environmental history and economic history are coming together to understand our predicament. This new field of "eco-economic history" traces the ecological impact of the startling rise in global economic productivity over the last two centuries. No longer is nature treated as an externality and damage to non-renewable resources discounted. I identify four basic eco-economic models emerging in this literature. The one I call retro-modernist returns us to a Euro-centered world for both the problem's origins and its remedies. Three more convincing models, double-layered modernity, parallel modernities, and multi-scalar approach-

JULIA ADENEY THOMAS

University of Notre Dame, Notre Dame, Indiana, Estados Unidos

thomasjna@aol.com

Traducción: Amanda Sucar Warrener es, expand our understanding of how we arrived at this catastrophic juncture and what we might do about it.

KEYWORDS: Anthropocene, climate change, economic history, environmental history, Asia, Japan, China, Africa, development 
stán surgiendo nuevas conversaciones entre historiadores ambientales y económicos, pues ambos campos replantean su entendimiento de las interacciones entre humanos y naturaleza bajo la categoría de Antropoceno. Aunque este término aún no se ha hecho oficial por la Unión Internacional de Ciencias Geológicas, se ha utilizado al menos desde 2002 para designar la transformación de la Tierra desde el Holoceno, con una duración relativamente predecible de 11700 años, hasta un nuevo estado permanente e impredecible menos propicio para los seres humanos. ${ }^{1}$ El Antropoceno indica una "ruptura" irreversible con el pasado (Hamilton, 2016). La situación es tan severa y desesperada que los climatólogos pueden medir los efectos en nueve sistemas planetarios, incluyendo - pero no limitados a - el cambio climático atmosférico. ${ }^{2}$ Ante esta situación, tanto la historia económica como la ambiental confrontan retos conceptuales que alteran sus premisas y acercan los campos. Los historiadores ambientales, que se han centrado durante mucho tiempo en la manera en que los humanos dan forma a los ecosistemas, ahora deben lidiar con lo que significa que los humanos den forma a la Tierra. Del mismo modo, algunos historiadores económicos que se preocuparon por las materias primas supusieron, en general, que si una sustancia no era abundante, se podrían encontrar o inventar sustitutos de formas bastante predecibles. Con la llegada de la nueva comprensión de las operaciones de la humanidad que alteran la Tierra, algunos investigadores sostienen que la historia económica y la historia ambiental deben hermanarse. En resumen, bajo la categoría del Antropoceno está emergiendo algo así como un campo de la "historia eco-económica". Mi análisis de este campo distingue cuatro modelos. Considero que necesitamos oponernos a uno y explorar y expandir los demás.

1 Para una introducción sobre el término "Antropoceno", véase Crutzen (2002). Para un entendimiento de los procesos por medio de los cuales los geólogos expertos en estratigrafía delimitan una nueva época, véase Zalasiewicz et al. (2010).

2 Para una discusión sobre los límites planetarios, véanse Rockström et al. (2009); Steffen et al. (2015). 


\section{Cuatro modelos eco-económicos}

Para enmarcar mi análisis de las formas en que las historias ambientales y económicas están convergiendo, quiero comenzar por subrayar los dos supuestos alentadores compartidos por todos los autores que analizo. En primer lugar, sin importar cómo definen el Antropoceno, todos coinciden en que es el resultado devastador de las actividades económicas que transforman los sistemas de la Tierra de los que dependen humanos y otras formas de vida. Vincular directamente el daño ambiental con la actividad económica da un vuelco en el campo de la economía a la tendencia de tratar los recursos naturales y las emisiones de residuos como "externalidades". Sumar estos costos ambientales a menudo cambia drásticamente los cálculos previos de eficiencia y beneficio. Ya sea que la devastación ambiental mundial haya surgido de la agricultura moderna temprana comercializada, la producción industrial basada en quema de carbón o la cultura de consumo masivo, estos nuevos análisis eco-económicos dejan claro que las economías no pueden trascender las ecologías. El crecimiento infinito no es posible, la sustitución infinita de una fuente natural de recursos por otra o la sustitución de capital y tecnología por recursos alcanzará su límite de manera eventual (Albritton, 2014). En síntesis, nuestro mundo físico es finito. A pesar de que muchos, de hecho, la mayoría de los historiadores económicos, todavía imaginan, junto con Kenneth Lipartito (2016), que pueden "repensar la materialidad" y dejar de lado la historia ambiental, analistas económicos más perspicaces, como Maxine Berg (2007; 2010), Timothy Mitchell (2011), Prasannan Parthasarathi (2011), Kenneth Pomeranz (2001; en prensa), Jeffrey Sachs (2015) y Amartya Sen (2014), reconocen que las propiedades de las sustancias y los sistemas naturales son el núcleo de la "materialidad", sin el cual cualquier replanteamiento de la historia económica es imposible (Lipartito, 2016: 102). ${ }^{3}$
En segundo lugar, al definir agencia y construir narrativas, todos los historiadores eco-económicos sugieren, explícita o implícitamente, no sólo una manera de entender el pasado, sino también de enfrentar los desafíos del presente. Preguntarse qué personas, grupos y procesos nos impulsaron hacia la nueva época turbulenta del Antropoceno también es una manera de cuestionar cómo podríamos restablecer un espacio para vivir decentemente en el futuro. La esperanza de todos, en otras palabras, es encontrar un mínimo de bienestar para nuestra especie, aun cuando los mares se elevan, las temperaturas se disparan, los océanos se acidifican, el aire contiene menos oxígeno, las especies no humanas desaparecen, las poblaciones humanas se disparan y la supervivencia se torna más difícil. Para decirlo de otra manera, el impulso detrás de vincular la historia económica y el medio ambiente es político en el sentido más amplio del término: el deseo de comprender de qué manera la distribución del poder y los recursos se desarrollan en un planeta finito. El resurgimiento reciente de la historia económica, después de su eclipse en la década de 1980, y su mayor compromiso con las cuestiones ambientales es ciertamente un muy buen desarrollo.

Dicho esto, algunos análisis son mejores que otros. Es posible distinguir cuatro configuraciones eco-económicas que cuentan la historia del desarrollo humano y la depredación de manera distinta. Tengo una forma abreviada de llamar a estas configuraciones eco-económicas. La primera, a la que llamo "retromodernista", me provoca serias dudas. Me refiero a las otras tres como "doble capa", "paralela" e "intersectada". Estos tres últimos modelos proporcionan una interpretación convincente de cómo la productividad económica y sus consecuencias transformaron el planeta.

3 Entre los historiadores económicos que continúan separando el crecimiento económico de su contexto material se incluye Joel Mokyr (2009), quien destaca la "economía del conocimiento". Para una revisión de la literatura sobre la Revolución industrial en Europa y el Antropoceno, véase Albritton (2012). 

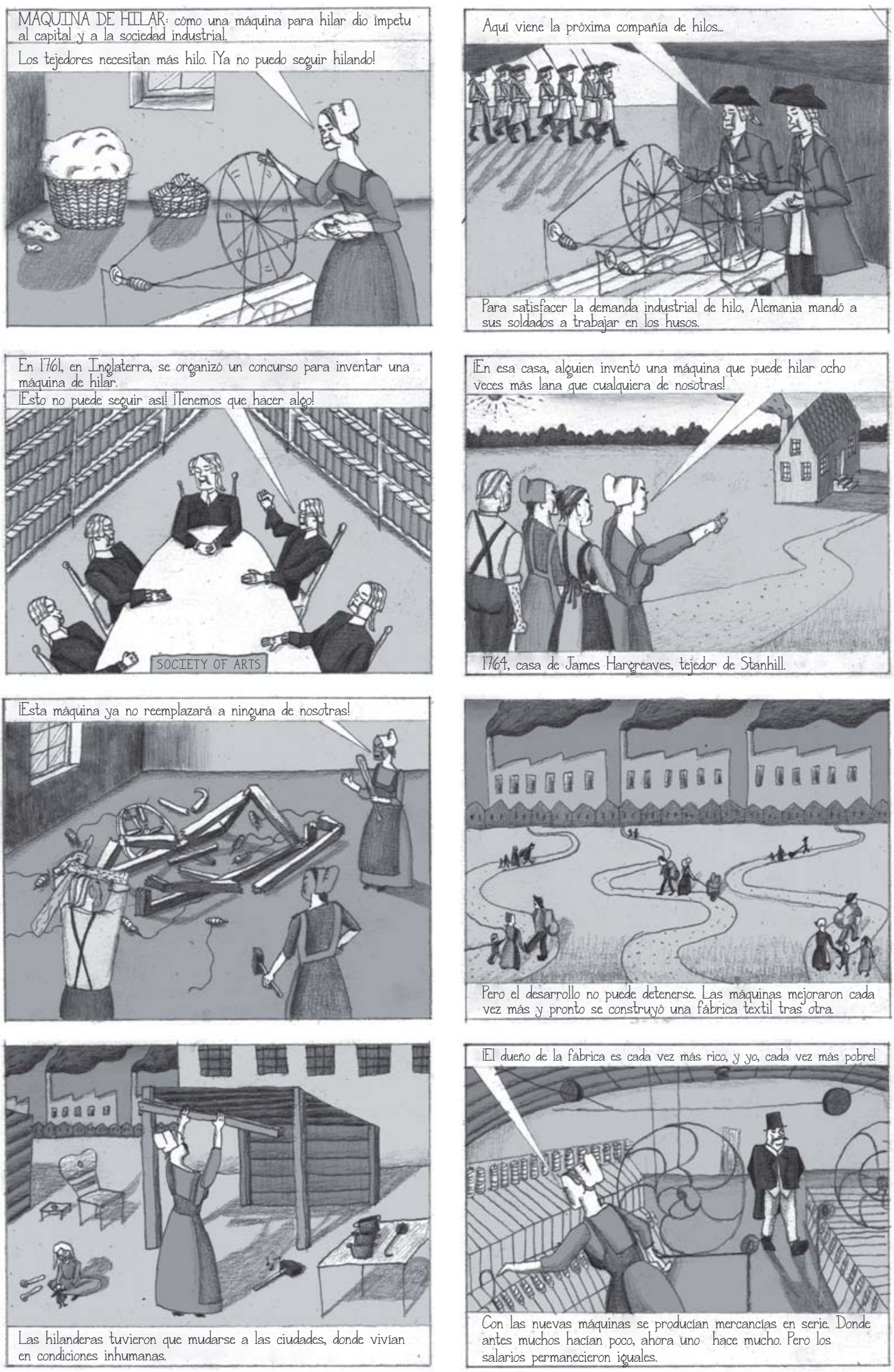


\section{EL MODELO RETROMODERNISTA}

\section{DEL CAPITALOCENO}

El primero de estos cuatro modelos eco-económicos, la postura retromodernista, culpa "al Occidente", sobre todo a la industrialización occidental y el desarrollo basado en combustibles fósiles, por los males del planeta. Muchos investigadores que trabajan en esta línea aparecen en la interesante Anthropocene Reading List de Verso Books. ${ }^{4}$ En general, atacan el término Antropoceno porque acusa de manera implícita a toda la especie humana y lo reemplazan con el término Capitaloceno o incluso Angloceno. Como sugieren estos términos alternativos, la narrativa nos regresa al norte de Europa de finales del siglo XVIII como el único origen de los procesos que transformaron el mundo. La culpa de la catástrofe inducida por el consumo de combustibles fósiles se asigna firmemente a un pequeño grupo de perpetradores. En la opinión de Andreas Malm y Alf Hornborg:

\footnotetext{
Una fracción infinitesimal de la población [...], una camarilla de hombres blancos británicos, literalmente utilizó la energía de máquinas de vapor como un arma (en mar y tierra, barcos y ferrocarril) contra la mejor parte de la humanidad, desde el delta del Níger hasta el delta del Yangtsé, del Levante a Latinoamérica” (2014: 64).
}

Uno no puede más que honrar el impulso de asignar responsabilidad por nuestro apresurado sufrimiento global. La matanza acelerada de otras especies y la destrucción en cámara lenta del bienestar humano nos convoca a la sala de juzgado del alma. "Justicia climática" es un grito frecuente en boca de la gente. ${ }^{5}$ Pero extrañamente, dado que las narrativas del Antropoceno deben involucrar al mundo entero, dado que las épocas geológicas, por definición, son fenómenos globales, entre los retromodernistas hay poco reconocimiento de la agencia de los pueblos y los procesos fuera del norte de Europa. Al igual que en la teoría de los sistemas mundiales de Wallerstein, tanto las personas no blancas como las ecologías no occidentales sirven principalmente como víctimas puras, mano de obra barata y materias primas. El viejo modelo difusionista de la tecnología surgida exclusivamente en Occidente hasta la década de 1850, cuando "alcanzó una escala global", se utiliza sin reconocer la investigación que desplaza esta narrativa de modernización eurocéntrica y que esta narrativa podría insinuar que todas las soluciones posibles deben venir también de Occidente (Bonneuil y Fressoz, 2016: 175). Además, se presta poca atención a la complejidad moral de la asignación de culpa por procesos que han durado 250 años y que causaron no sólo gran parte de lo que era malo o imperfecto, sino también importantes beneficios, como una mayor longevidad y mayor democracia. Por último, en el enfoque retromodernista la discusión sostenida sobre el crecimiento de la población mundial está casi ausente y la terrible depredación del medio ambiente de los estados comunistas no ha sido examinada.

Aún más problemático que identificar a los propietarios de fábricas del siglo XVIII de Manchester como la raíz de todas nuestras dificultades, es el ataque a los climatólogos por parte de algunos retromodernistas. The Shock of the Anthropocene (Bonneuil y Fressoz, 2016), por ejemplo, tiene como objetivo elaborar la definición "autorizada" de tres etapas del Antropoceno, comenzando hace 250 años. Esta definición "oficial” proclama supuestamente una "gran narrativa" de científicos heroicos y nos alerta del peligro de un objetivo de dominio mundial. Es difícil saber cómo tomar esta afirmación. En primer lugar, no existe un Antropoceno

\footnotetext{
$4 \quad$ Véanse, en particular, Wark (2015); Moore (2015); Malm (2016); Bonneuil y Fressoz (2016).

$5 \quad$ Véanse, por ejemplo, Chancel y Piketty (2015); Raworth (2013).
} 
"oficial" o "autorizado". Los geólogos siguen discutiendo categóricamente su definición y duración, y continuarán haciéndolo en los próximos años, incluso después del voto unánime del 16 de agosto de 2016 del Grupo de Trabajo del Antropoceno (AGW, por sus siglas en inglés), ${ }^{6}$ que es el paso preliminar en un proceso de múltiples etapas. En segundo lugar, Bonneuil y Fressoz advierten de una "exaltación exagerada de los conocimientos científicos actuales", pero dado que los climatólogos son objeto de abuso personal, que su trabajo no tiene fondos suficientes, su correo electrónico es invadido y sus descubrimientos menospreciados o ignorados, esto difícilmente podría parecer un problema apremiante (2016: 79). Por último, hay poca evidencia de la afirmación de que un grupo de científicos está planeando dominar el mundo. Es cierto que el químico Paul Crutzen, quien acuñó el término Antropoceno junto con Eugene Stoermer, argumenta que los científicos y los ingenieros deben "orientar a la sociedad hacia una gestión ambientalmente sostenible" (2002: 23), pero “orientación" y "gobernanza" no son sinónimos. De hecho, la alarma sobre un llamado "geogobierno de los científicos" parece fuera de lugar cuando los políticos que niegan el cambio climático, los tecnogurús y los imprudentes capitalistas de riesgo están a la mano en todas partes. En resumen, el análisis retromodernista no es convincente geológica, histórica, ni políticamente (Bonneuil y Fressoz, 2016: 79).

\section{MODELO DE DOBLE CAPA}

\section{DEL CRECIMIENTO MODERNO}

Mientras la teoría de los sistemas mundiales puede estar recuperando popularidad entre algunos historiadores económicos europeos preocupados por el Antropoceno, los historiadores especializados en Asia, África y Latinoamérica están profundamente conscientes de que este modelo de procesos de modernización de arriba abajo a escala planetaria oscurece lo que de manera cariñosa y falsa se denomina prácticas "tradicionales", que se encuentran por debajo. El punto central es que, en muchos casos, las prácticas económicas occidentales globalizadas habrían fallado si no hubieran sido transformadas por técnicas y tecnologías de producción locales más en sintonía con las especificidades ecológicas. Ambas capas de desarrollo, la superior y la inferior, se combinaron para impulsarnos hacia el Antropoceno. Aunque previamente fueron pasados por alto o despreciados los modos locales de producción económica, atraen ahora la atención de la historia ambiental y económica precisamente porque están inmersos en dinámicas de retroalimentación ecológica más complejas. Esta posición hace que sea posible tomar en serio el uso revolucionario de la energía del carbón en Gran Bretaña, como lo demuestra E. A. Wrigley (2010), sin convertirlo en el progenitor exclusivo de la transformación del sistema de la Tierra. Los empresarios de la energía de China, como Liu Hongsheng (1888-1956), necesariamente forman parte de la historia mundial (Muscolino, 2016).

Por ejemplo, el historiador ambiental Corey Ross (en prensa) ha proporcionado una elegante exposición de la compleja relación entre occidentalistas que funcionan en la escala mundial y los pequeños agricultores en el sudeste asiático. Estos dos grupos, como señala el autor, pueden distinguirse como los que están en el asiento del conductor del Antropoceno y los que se sentaron en la parte trasera hasta la "gran aceleración" de mediados del siglo XX, pero ambos contribuyeron a los procesos extractivos que alteraron el planeta por medio de la producción de caucho. ${ }^{7}$ A principios del siglo Xx,

6 Para una explicación de este proceso, véase Zalasiewicz et al. (2015).

7 Sobre el concepto de "gran aceleración", véase Steffen, crutzen y McNeill (2007). 
magnates del caucho comercial y sus aliados imperialistas sentían que llevaban el peso de la historia sobre sus hombros mientras marchaban hasta Malasia, Indonesia, y más adelante, Tailandia. Plantar sus árboles de caucho en líneas ordenadas y racionalizar la producción les parecía axiomáticamente mejor. A su modo de pensar, una mayor abstracción debía conducir seguramente a una mayor extracción. Dieron por hecho que el dinámico sector europeo orientado a la exportación superaría el rendimiento de la producción de un pueblo indígena. El caucho, sin embargo, goteó mejor de los árboles alimentados en ecosistemas complejos que de aquellos en líneas rectas. Como explica Ross (en prensa), cuando los árboles de caucho de los pequeños agricultores produjeron más caucho y resultaron menos susceptibles a la enfermedad, los plantadores coloniales se mostraron incrédulos y asombrados. Desde su perspectiva, era sorprendente que personas que se suponía que eran ineficaces como agentes económicos - y estéticamente ofensivos en el cuidado de la simetría ordenada al momento de plantar sus cosechas- resultaron capaces de extraer más de su entorno natural. La proporción de caucho cultivado en parcelas aumentó de forma constante de la década de 1910 en adelante y con el tiempo igualó o superó la producción de las fincas en los principales países de exportación del sudeste asiático (Ross, en prensa). En contraste con el pensamiento retromodernista, las eco-economías de menor escala, incrustadas en desorden en las redes ecológicas locales, eran más adecuadas para el objetivo de producir un bien comercial fundamental para crear el Antropoceno. Si el conocimiento global y el local no se

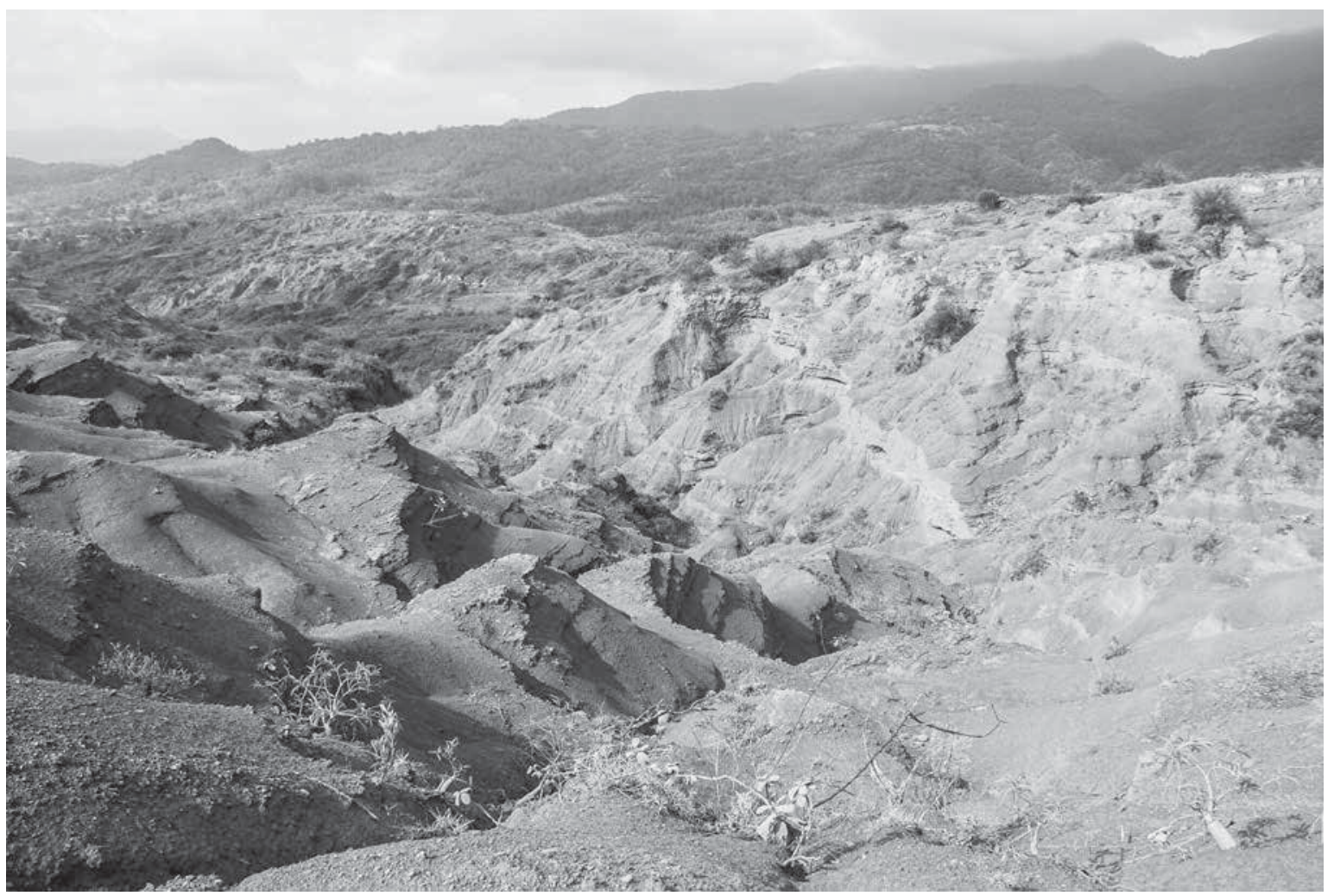

Pavel LaRa LozAda • La tala desmedida ha causado daños irreparables al ambiente en Santa María Tiltepec, Oaxaca, 2015. 
hubieran fusionado, tal vez no estaríamos encaminados hacia una catástrofe ambiental como lo estamos en la actualidad.

Desde el punto de vista político y ético, el resultado de un análisis que toma en cuenta ambas capas de crecimiento moderno es que las acusaciones de culpabilidad y protestas de inocencia se enturbian. Por ejemplo, en The Great Derangement - El gran desorden-, Amitav Ghosh (2016) sugiere de manera intrigante que la desigualdad del desarrollo capitalista podría incluso haber retrasado el incremento de los gases de efecto invernadero procedentes de Asia, debido a que la industrialización de este continente se demoró por el colonialismo occidental. Además, la evaluación de los beneficios y daños del desarrollo global también adquiere complejidad. Por ejemplo, la combinación de iniciativas de saneamiento, erradicación de enfermedades y aumento de la productividad agrícola de la revolución verde —en el sur de Asia, aunque no en África— produce poblaciones humanas más sanas, pero también ha dado lugar a un aumento sin precedentes de las tasas de crecimiento de la población y de los números totales: 1500 millones de personas habitaban la Tierra alrededor de 1900; 3000 millones en la década de 1960 y más de 7500 millones en la actualidad. Como señala el historiador económico Tirthankar Roy, "el crecimiento (humano) de la población ha sido malo para el medio ambiente" (en prensa). Sin embargo, por fortuna, así como las causas económicas del Antropoceno se hacen más numerosas con el modelo de doble capa, también lo hacen las mejoras potenciales. Los megaproyectos de geoingeniería, tan amados por los ecomodernistas y el Banco Mundial, ya no parecen soluciones inevitables. ${ }^{8}$ Aunque nunca podremos volver a las condiciones del Holoceno e incluso su memoria se pierde, ya que "ninguna persona menor de 30 años ha vivido un mes de temperatura global por debajo del promedio del siglo XX" (Gillis, 2015), la comprensión de la variedad y el arraigo ecológico de las prácticas productivas puede ayudar a imaginar economías políticas alternativas y sostenibles (Gillis, 2015).

\section{MODERNIDADES PARALELAS}

Una tercera forma de pensar en la eco-economía es sugerir estrategias de desarrollo paralelas, con fortalezas en varias regiones. En lugar de las abrumadoras actividades microescalares "retrógradas" del modelo Capitaloceno de desarrollo occidental de los retromodernistas, o el modelo de doble capa centrado en la interacción de los sistemas globales y locales de producción, otra forma de concebir la historia de las economías y los ambientes es reconocer varias modernidades viables y coexistentes que emergen más o menos al mismo tiempo. El concepto de modernidades múltiples se basa en el reconocimiento de que los ambientes naturales provocaron vías de desarrollo distintas, pero viables. Como ha indicado la reciente investigación de los historiadores económicos Kenneth Pomeranz (2001) y Prasannan Parthasarthi (2011), ante la fuerza aplastante del imperialismo europeo del siglo XIX, las zonas no occidentales eran más dinámicas de lo que suele suponerse, y en algunos lugares, como Japón, esta vitalidad económica continúa en la actualidad. ${ }^{9}$ Las tres principales distinciones entre las modernidades del desarrollo de Occidente y no Occidente podrían resumirse así: en contraste con el desarrollo intensivo del capital de Europa, se encuentra el desarrollo intensivo de la mano de obra de Asia oriental; en oposición a los regímenes intensivos de la tierra en Europa y partes de Asia, existe la agricultura extensiva de la tierra en África; contra los regímenes de uso de alta energía de importantes estados

$8 \quad$ Para "ecomodernismo", véanse Asafu-Adjaye et al. (2015); Shellenberger y Nordhaus (2005)

9 Véanse, por ejemplo, Pomeranz (2001); Minichiello (1998). 
capitalistas y comunistas —en particular, Estados Unidos y la Unión Soviética-, se encuentra la eficiencia energética de Japón, Corea del Sur y Taiwán.

El entendimiento de que hay varias vías para el desarrollo de sociedades humanas sanas y seguras ofrece más opciones al considerar las respuestas aceptables para el Antropoceno. El historiador económico Kaoru Sugihara (1996) ha argumentado que la vía de desarrollo de Asia oriental, que ha sido menos intensiva en recursos que la de la mayoría de los países del Atlántico Norte, ofrece más esperanza para el desarrollo sostenible. Los datos de Japón, Taiwán y Corea del Sur dan una explicación plausible a esa esperanza. Aprenderemos más si eliminamos por completo la visión retromodernista que supone que la modernidad occidental industrial -intensiva en capital, con régimen intensivo de la tierra y uso de alta energía - era la única vía posible para el bienestar. Otros modelos de actividad económica, más acordes con la supervivencia ecológica en un planeta con recursos finitos, pueden, sin embargo, detectarse en el mundo no occidental, cuando las historias ambientales y ecológicas se reúnen.

Por ejemplo, la predilección por los procesos intensivos de mano de obra y recursos en el sector industrial del Japón moderno tiene una larga historia. Los principios de la frugalidad articulados al comienzo del Japón moderno abogaron de manera explícita por la maximización del uso eficiente de los recursos limitados, incluso cuando implicara mayor uso de mano de obra humana. Después de la industrialización de Japón, las autoridades locales continuaron en esta línea y lideraron el camino en temas de conservación de energía y reducción de humo. Como ha argumentado el historiador económico Kobori Satoru, los industriales se sentían atraídos por las técnicas de fabricación de conservación de energía en la década de 1920, cuando Japón ya no era autosuficiente en carbón y los precios habían aumentado. En las "crisis del petróleo" de la década de 1970, Japón estaba preparado para hacer mejoras adicionales, y en 1990, era el líder mundial en eficiencia energética. Estos éxitos se debieron a patrones históricos y culturales, así como a los preceptos del mercado y las limitaciones de recursos. La comprensión de diversas vías regionales como historias de efectos de retroceso igualmente viables retratan una sola trayectoria global para el bienestar o la ruina.

\section{ESCALAS INTERSECTADAS}

La cuarta y última configuración de las escalas ecoeconómicas considera la multidimensionalidad de factores en juego en cualquier situación. En especial con la "gran aceleración" de la población humana y su explotación de los recursos naturales desde la Segunda Guerra Mundial, el éxito en un ámbito a menudo viene acompañado del fracaso en otro, debido a que múltiples marcos de tiempo, escalas espaciales y diferentes tipos de agentes chocan dentro de márgenes de error ecológicos cada vez más estrechos. Solucionar un problema puede exacerbar otro. Por ejemplo, la fabricación de acero basada en un proceso de oxígeno básico en Fuji Iron Works conservó la energía pero empeoró la contaminación del aire en la década de 1950, y ver el Monte Fuji desde el centro de Tokio pronto fue imposible.

La energía nuclear proporciona un ejemplo dramático de la complejidad de los problemas actuales. El economista Amartya Sen señaló recientemente que "hay al menos cinco tipos diferentes de factores externos que se suman de manera significativa a los costos sociales de la energía nuclear" (2014). Dado que estos costos no pueden ser medidos por los mercados o por análisis de costobeneficio convencionales y se extienden a lo largo del tiempo en escalas muy distintas - como en la vida media del uranio-, y en el espacio - como en los residuos nucleares de Japón enviados a Suecia-, Sen argumenta que deben utilizarse estimados de 
probabilidad para considerar el impacto de la energía nuclear:

A pesar de las pequeñas probabilidades de cada uno de estos peligros, la suma de los cinco, multiplicada por el número cada vez mayor de empresas nucleares, en general tiende a producir probabilidades considerables. Las estimaciones de daño probable (de terrible a catastrófica) podrían ser gigantescas (2014).

La necesidad de desarrollar un marco normativo que contemple todas estas dimensiones es crucial. Como concluye Sen: "el pensamiento ambiental tiene que ser multidireccional en lugar de centrado en un solo objetivo, aun cuando ese objetivo sea algo tan importante como la amenaza climática de las emisiones de carbono" (2014). China es ejemplo clásico cuando se trata de ejemplificar la dificultad de satisfacer toda la gama de objetivos necesarios para el bienestar humano. Como Kenneth Pomeranz ha evidenciado, la solución de un problema enorme - limitar las emisiones de energía- podría generar otro problema de igual dimensión - la escasez de agua, que es peor-. En la actualidad, la gente en China sufre escasez de agua:

El agua superficial y cerca de la superficie per cápita en China hoy es más o menos una cuarta parte de la media mundial; peor aún, es distribuida de manera muy desigual. El norte y noroeste de China, con casi 30\% de la población nacional y más de la mitad de la tierra cultivable del país, tienen alrededor de $7 \%$ de su agua superficial; por lo tanto, sus recursos hídricos superficiales per cápita son de 20 a 25\% de la media de China en su conjunto, o de 5 a $6 \%$ de la media global (Pomeranz, 2017: 279).

Para resolver este problema, podrían hacerse algunas intervenciones locales para la eficiencia del recurso - como la reparación de tuberías con fugas-, pero incluso la reparación de todas las tuberías con fugas y el uso más eficiente del agua no proporcionarían suficiente líquido para satisfacer las necesidades crecientes de China. Sin embargo, las medidas a gran escala para encontrar y distribuir más agua elevarían las emisiones energéticas de China y contaminarían su aire. Los megaproyectos de ingeniería que se han propuesto incluyen el tratamiento de aguas residuales, la desalinización y el gigantesco desvío Sur-Norte, que implicaría el bombeo de agua a lo largo de miles de kilómetros. Todo exigiría un gran incremento de gases de efecto invernadero. Al parecer, el problema se reduce a que China tenga agua potable o aire respirable, pero no ambos. Con la reducción dramática del tiempo disponible para la introducción de tecnologías alternativas a medida que se acelera el Antropoceno, debemos hacer frente a la posibilidad de que los problemas del crecimiento son inextricables y que la única manera de garantizar "la sostenibilidad con decencia" es centrarse en los modelos económicos de decrecimiento o en el mejor estado estacionario (Thomas, 2011). Cuando la historia económica y la ambiental convergen, a menudo el resultado no es una historia feliz.

\section{Conclusión}

La terrible noticia es que los sistemas planetarios están dando patadas como un semental enloquecido, sorprendiendo incluso a los climatólogos más atentos. El aumento de las concentraciones de $\mathrm{CO}_{2}$ en 2015 fue el mayor anual registrado y el $\mathrm{CO}_{2}$ ha alcanzado un nivel no visto en 15 millones de años —405.6 partes por millón-. El climatólogo Michael Mann afirma que "no nos queda presupuesto de carbono para la meta de $1.5^{\circ} \mathrm{C}$ y la oportunidad para mantener los $2{ }^{\circ} \mathrm{C}$ está desapareciendo rápidamente, a menos que el mundo comience a reducir las emisiones con determinación comenzando ahora mismo" (citado en Hopkins, 2016). La producción de nitrógeno, el calentamiento de los océanos y 
la sexta gran extinción de especies no ha disminuido. Por estas razones, celebro el compromiso entre las historias económicas y ambientales, pero señalo las deficiencias del paradigma retromodernista, en el cual la responsabilidad de crear y confrontar el Antropoceno recae sólo sobre "el Occidente". Quizá proporciona consuelo el pensar que podría haber un grupo de capitalistas y científicos "en el puesto de mando de un planeta desaliñado y su humanidad errante" (Bonneuil y Fressoz, 2016: 80), pero la dinámica anticuada del opresor discreto contra quien los rectos pueden rebelarse podría ser justo otro de esos poderosos tropos modernistas - como el crecimiento infinito- que nuestras circunstancias radicalmente alteradas nos obligan a abandonar. Es necesario apuntar hacia una nueva comprensión para crear una estrategia para nuestra supervivencia con base en interconexiones, atenta a los matices, flexible y generosa, porque en diferentes momentos y en diferentes formas todos emergemos como testigos, víctimas y perpetradores en este nuevo mundo turbulento. Los lugares que solían ser descartados como "tardíos" o "retrasados" en su desarrollo podrían de hecho brindar mejores perspectivas sobre cómo vivir sencillamente para que todos vivan. ${ }^{10}$ Asia, quizá más que cualquier otra región, debido a su tamaño, población y variadas condiciones económicas y ambientales, emerge como un ejemplo crucial para los historiadores eco-económicos del Antropoceno y para cualquier posible reconciliación. Afortunadamente, tres formas alternativas de entender las fuerzas económicas que han dado forma a nuestro mundo han surgido en la literatura. Si bien el modelo a escala de doble capa, el paralelo o el de intersección no producen buenas noticias absolutas, los tres arrojan luz sobre el problema y nuestras posibilidades.

10 Véase, por ejemplo, Shrestha (2016).

\section{Bibliografía}

Albritton Jonsson, Fredrik, 2012, "The Industrial Revolution in the Anthropocene”, en Journal of Modern History, vol. 84, núm. 3, pp. 679-696.

__ 2014, "The Origins of Cornucopianism: A Preliminary Genealogy", en Critical Historical Studies, vol. 1, núm. 1, pp. 151-168.

Asafu-Adjaye, John et al., 2015, "An Ecomodernist Manifesto". Disponible en línea: <http://www.ecomodernism.org/manifesto-english/>.

Berg, Maxine, 2007, Luxury and Pleasure in Eighteenth Century Britain, Oxford University Press, Oxford.

— , 2010, "The British Product Revolution of the Eighteenth Century", en Jeff Horn, Leonard N. Rosenband y Merritt Roe Smith (eds.), Reconceptualizing the Industrial Revolution, Miт Press, Massachusetts, pp. 47-64. Disponible en línea: <http://www.jstor.org/stable/j. ctt5hhgdm.7>.

Bonneuil, Christophe y Jean-Baptiste Fressoz, 2016, The Shock of the Anthropocene: The Earth, History, and Us, Verso Books, Nueva York. Chancel, Lucas y Thomas Piketty, 2015, "Carbon and Inequality: From Kyoto to Paris", reporte presentado en la conferencia "Environmental Inequalities", Institute for Sustainable Development and International Relations y The Paris School of Economics, 3 de noviembre, París. Crutzen, Paul J., 2002, “Geology of Mankind”, en Nature, vol. 415, núm. 6867, p. 23.

Ghosh, Amitav, 2016, The Great Derangement: Climate Change and the Unthinkable, University of Chicago Press, Chicago.

Gillis, Justin, 2015, "Climate Accord is a Healing Step, if Not a Cure", en The New York Times, 12 de diciembre. Disponible en línea: <https://www. nytimes.com/2015/12/13/science/earth/climate-accord-is-a-healing-step-if-not-a-cure.html?_r=1>. Consultado el 13 de diciembre de 2016.

Hamilton, Clive, 2016, "The Anthropocene as Rupture”, en The Anthropocene Review, vol. 3, núm. 2, pp. 93-106.

Hopkins, Rob, 2016, "Budget 2016: What we Are Facing Isn't a Financial Crisis, but a Crisis of the Imagination”, en Transition Network, 16 de marzo. Disponible en línea: <https://www.transitionnetwork.org/blogs/rob-hopkins/2016-03/budget-2016-what-we-are-facing-isntfinancial-crisis-crisis-imagination>. Consultado el 17 de marzo de 2016.

Kobori, Satoru, 2017, "The Development of Energy Conservation Technology in Japan, 1920-70: An Analysis of Energy-Intensive Industries and Energy-Conservation Policies", en Gareth Austin (ed.), Economic Development and Environmental History in the Anthropocene: Perspectives on Africa and Asia, Bloomsbury Academic, Londres. 
Lipartito, Kenneth, 2016, "Reassembling the Economic: New Departures in Historical Materialism”, en American Historical Review, vol. 121, núm. 1, pp. 101-139.

Malm, Andreas, 2016, Fossil Capital: The Rise of Steam-Power and the Roots of Global Warming, Verso Books, Nueva York.

Malm, Andreas y Alf Hornborg, 2014, "The Geology of Mankind? A Critique of the Anthropocene Narrative", en The Anthropocene Review, vol. 1, núm. 1, pp. 62-69.

Metzler, Mark, 2013, Capital as Will and Imagination: Schumpeter's Guide to the Postwar Japanese Miracle, Cornell University Press, Ithaca.

Minichiello, Sharon, 1998, Japan's Competing Modernities: Issues in Culture and Democracy, 1900-1930, University of Hawaii Press, Honolulu. Mitchell, Timothy, 2011, Carbon Democracy: Political Power in the Age of Oil, Verso Books, Londres.

Mokyr, Joel, 2009, The Enlightened Economy: An Economic History of Britain, 1700-1850, Yale University Press, New Haven.

Moore, Jason W., 2015, Capitalism in the Web of Life: Ecology and the Accumulation of Capital, Verso Books, Nueva York.

Muscolino, Micah, 2016, "Energy and Enterprise in Liu Hongsheng's Cement and Coal-Briquette Businesses, 1920-1937”, en Twentieth-Century China, vol. 41, núm. 2, pp. 159-179.

Nordhaus, Ted y Michael Shellenberger, 2007, Break Through: From the Death of Environmentalism to the Politics of Possibility, Houghton Mifflin Harcourt, Boston.

Parthasarathi, Prasannan, 2011, Why Europe Grew Rich and Asia Did Not: Global Economic Divergence, 1600-1850, Cambridge University Press, Cambridge.

Pomeranz, Kenneth M., 2001, The Great Divergence: China, Europe, and the Making of the Modern World Economy, Princeton University Press, Nueva Jersey.

—_, 2017, "Water, Energy, and Politics: Chinese Industrial Revolutions in Environmental Perspective”, en Gareth Austin (ed.), Economic Development and Environmental History in the Anthropocene: Perspectives on Africa and Asia, Bloomsbury Academic, Londres.

Raworth, Kate, 2013, "Defining a Safe and Just Space for Humanity", en State of the World 2013: Is Sustainability Still Possible?, Island Press, Washington, D. C., pp. 28-38.

Rockström, Johan et al., 2009, "Planetary Boundaries: Exploring the Safe Operating Space for Humanity", en Ecology and Society, vol. 14, núm. 2, artículo 32.

Ross, Corey, en prensa, "Developing the Rainforest: Rubber, Environment and Economy in Southeast Asia”, en Gareth Austin (ed.), Economic Development and Environmental History in the Anthropocene. Perspectives on Asia and Africa, Bloomsbury, Londres.

Roy, Tirthankar, en prensa, "Land Quality, Carrying Capacity, and Sustainable Agricultural Change in 20th-Century India", en Gareth Austin (ed.), Economic Development and Environmental History in the Anthropocene. Perspectives on Asia and Africa, Bloomsbury, Londres.

Sachs, Jeffrey, 2015, The Age of Sustainable Development, Columbia University Press, Nueva York.

Sen, Amartya, 2014, "Global Warming Is Just One of Many Environmental Threats that Demand Our Attention", en New Republic, 22 de agosto. Disponible en línea: <http://www.newrepublic.com/article/118969/environmentalists-obsess-about-global-warming-ignore-poorcountries>. Consultado el 22 de agosto de 2015.

Shellenberger, Michael y Ted Nordhaus, 2005, The Death of Environmentalism: Global Warming Politics in a Post-Environmental World, The Breakthrough Institute, Oakland.

Shrestha, Shail, 2016, "The Paris Climate Talks: A Nepali View", en Local Futures. Economics of Happiness, 15 de marzo. Disponible en línea: <http://www.localfutures.org/climate-talks-a-nepali-view>. Consultado el 17 de marzo de 2016.

Steffen, Will et al., 2015, "Planetary Boundaries: Guiding Human Development on a Changing Planet”, en Science, vol. 347, núm. 6223.

Steffen, Will, Paul J. Crutzen y John R. McNeill, 2007, “The Anthropocene: Are Humans Now Overwhelming the Great Forces of Nature?”, en Ambio, vol. 36, núm. 8, pp. 614-621.

Sugihara, Kaoru, 1996, "The European Miracle and the East Asian Miracle: Towards a New Global Economic History", en Sangyo to Keizai, vol. 11, núm. 2, pp. 27-48.

Thomas, Julia Adeney, 2011, "From Modernity with Freedom to Sustainability with Decency: Politicizing Passivity", en Kimberly Coulter y Christof Mauch (eds.), The Future of Environmental History: Needs and Opportunities, Rachel Carson Center for Environment and Society (Perspectives 3), Múnich, pp. 53-67.

Wark, McKenzie, 2015, Molecular Red: Theory for the Anthropocene, Verso Books, Nueva York.

Wrigley, E. A., 2010, Energy and the English Industrial Revolution, Cambridge University Press, Cambridge.

Zalasiewicz, Jan, Mark Williams, Will Steffen y Paul Crutzen, 2010, "The New World of the Anthropocene", en Environmental Science and Technology, vol. 44, núm. 7, pp. 2228-2231.

Zalasiewicz, Jan et al., 2015, "When did the Anthropocene Begin? A Mid-Twentieth Century Boundary Level is Stratigraphically Optimal”, en Quaternary International, vol. 383, pp. 196-203. 